\title{
Effects of Dust on Gravitational Lensing by Spiral Galaxies
}

\author{
Rosalba Perna and Abraham Loeb \\ Harvard-Smithsonian Center for Astrophysics, MS-51 \\ 60 Garden Street, Cambridge MA 02138 \\ Matthias Bartelmann \\ Max-Planck-Institut für Astrophysik \\ Karl-Schwarzschild-Strasse 1, D-85748 Garching, Germany
}

\begin{abstract}
Gravitational lensing of an optical QSO by a spiral galaxy is often counteracted by dust obscuration, since the line-of-sight to the QSO passes close to the center of the galactic disk. The dust in the lens is likely to be correlated with neutral hydrogen, which in turn should leave a Ly $\alpha$ absorption signature on the QSO spectrum. We use the estimated dust-to-gas ratio of the Milky-Way galaxy as a mean and allow a spread in its values to calculate the effects of dust on lensing by low redshift spiral galaxies. Using a no-evolution model for spirals at $z \lesssim 0.8$ we find, in $\Lambda=0$ cosmologies, that the magnification bias due to lensing is stronger than dust obscuration for QSO samples with a magnitude limit $B \lesssim 16$. The density parameter of neutral hydrogen, $\Omega_{\mathrm{HI}}$, is overestimated in such samples and is underestimated for fainter QSOs.
\end{abstract}

Subject headings: cosmology: theory - gravitational lensing — quasars: absorption lines

ApJ, in press, 1997 


\section{Introduction}

Gravitational lensing by spiral galaxies results in multiple images with sub-arcsecond separation. A spiral galaxy at a cosmological distance would lens a background QSO if the line-of-sight to the QSO passed within a few kpc from the disk center. Under these circumstances, the neutral hydrogen in the disk would inevitably leave a strong Ly $\alpha$ absorption signature on the quasar spectrum. A significant HI column density was indeed inferred for the spiral galaxy lens B0218+357 which has an Einstein ring radius 0." 3 (Carrilli et al. 1993).

Strong Ly $\alpha$ absorption is expected to result even at large impact parameters from the centers of galactic disks ( $\sim 10-20 \mathrm{kpc})$, where the effect of lensing is weak. In fact, damped Ly $\alpha$ absorption systems with neutral hydrogen column densities $\gtrsim 10^{20} \mathrm{~cm}^{-2}$ are identified routinely in the spectra of high redshift QSOs, with an abundance consistent with that expected for the progenitors of present-day disk galaxies (Wolfe 1995). The association between damped Ly $\alpha$ absorbers and the progenitors of present-day disk galaxies is supported by several observational facts. First, the abundance of heavy elements at low ionization stages in the absorbers and the velocity field traced by them is consistent with values expected for disk galaxies (Turnshek et al. 1989; Wolfe et al. 1993; Lu et al. 1993; Pettini et al. 1994; Lu \& Wolfe 1994). Recent Keck HIRES observations by Wolfe (1995; see also Lanzetta \& Bowen 1992) indicate that the weak low-ionization metal absorption lines in these systems often show the highest column-density component at either the red or the blue edge of the velocity profile. Such an asymmetry is characteristic of absorption by a rotating gaseous disk (Wolfe 1995). Second, observations of redshifted 21-cm absorption and emission from damped Ly $\alpha$ absorbers indicate disk-like structures of galactic dimensions (Briggs et al. 1989; Wolfe et al. 1992), and Faraday-rotation observations are consistent with the existence of micro-Gauss magnetic fields in these systems (Wolfe, Lanzetta, \& Oren 1992; Welter, Perry, \& Kronberg 1984; Perry, Watson, \& Kronberg 1993). Finally, the association of the damped Ly $\alpha$ absorbers with star-forming galaxies is directly confirmed by direct imaging (Steidel et al. 1994, 1995) in most of the cases. However, whereas some systems are clearly spirals, others show irregular morphologies (Le Brun et al. 1996). For simplicity, we focus only on spirals. A more general treatment of this problem would involve many more free parameters and is beyond the scope of this paper. Neverthless, we stress that if dwarf galaxies with relatively shallow potential wells make a significant fraction of all damped absorbers, then our analysis overestimates the importance of lensing.

The mass density of neutral hydrogen at $z \approx 3, \Omega_{\mathrm{HI}} \approx 0.005$, is comparable to the mass density in stars today, and appears to steadily decline at lower redshifts (Lanzetta, Wolfe, \& Turnshek 1995; Wolfe 1995 and references therein). The simplest interpretation of this result is that the material in present-day disk galaxies had already assembled at a redshift $z \approx 3$, while the observed gradual decline of $\Omega_{\mathrm{HI}}(z)$ since then is simply a result of the consumption of the gas by star formation.

Based on these indications and the evidence that the velocity width of the associated metal 
lines is indicative of rotational velocities $\sim 200 \mathrm{~km} \mathrm{~s}^{-1}$ (Wolfe 1995), Bartelmann \& Loeb (1996) have adopted the view that damped Ly $\alpha$ absorption in QSO spectra results from neutral hydrogen disks in the present-day potential wells of spiral galaxies. Following this assumption, they showed that damped Ly $\alpha$ systems may lens their background QSOs. An important consequence thereof is that the statistics of the absorbers themselves, as inferred from the number and distribution of absorption troughs detected, can be affected by the magnification bias. Because of the magnification of their flux due to lensing, the number of QSOs observed above a given magnitude threshold is increased, and consequently the observed fraction of QSOs which show damped Ly $\alpha$ absorption is enhanced.

An effect which tends to counteract the magnification by lensing is obscuration of the source by dust. By now, there is evidence that damped Ly $\alpha$ systems contain measurable quantities of dust. A dust-to-gas ratio of order $10 \%$ of the Milky-Way value is inferred from the enhanced level of reddening found in a sample of QSOs that have damped Ly $\alpha$ absorption (Fall, Pei, \& McMahon 1989; Pei, Fall, \& Bechtold 1991), and from the gas-phase depletion of Cr relative to Zn (see Meyer, Welty, \& York 1989; Meyer \& Roth 1990; Pettini, Boksenberg, \& Hunstead 1990; Meyer \& York 1992; Pettini et al. 1994; Wolfe et al. 1994; Smith et al. 1996; although Lu, Sargent, \& Barlow (1996) challenge this view based on the abundances of other metals). By dimming the light of the background sources, dust reduces the number of QSOs above a given magnitude threshold (Ostriker \& Heisler 1984; Fall \& Pei 1988, 1993), thus opposing the effect of the magnification bias due to lensing. It is thus a relevant issue to consider these two effects simultaneously and to estimate their relative importance. This is the purpose of the present paper. The detection of lensing by low-redshift spiral galaxies, such as B0218+357 at $z=0.68$ (O'Dea et al. 1992; Patnaik et al. 1993), 2237+0305 at $z=0.04$ (Huchra et al. 1995), and B1600+434 (Jaunsen \& Hjorth 1997) implies that dust obscuration does not mask lensing altogether.

The efficiency of gravitational lensing is expected to strongly peak in the redshift interval $0.2 \lesssim z \lesssim 0.8$. Aside from the tendency of cosmological lenses to maximize their influence around $z \sim 0.5$ (Schneider, Falco, \& Ehlers 1992; Bartelmann \& Loeb 1996), evolutionary phenomena such as the hierarchical merging of halos and the HI consumption by star formation, would tend to reduce the significance of lensing per HI column density at high redshifts. We therefore focus in this paper only on spiral lenses at low-redshifts $z \lesssim 0.8$. At these redshifts, there are various indications that the potential wells of spirals did not evolve significantly due to merging:

1. Substantial merging or accretion would have resulted in asymmetric distortion of the isophotes of spiral galaxies. Direct observations of the level of lopsidedness in spiral galaxies set an upper limit on the current accretion rate of companions with a mass of order $10 \%$ the parent galaxy mass, which is 0.25 companions per Gyr (Zaritzky \& Rix 1996). This implies that the fractional increase in the mass of galactic disks due to major mergers is smaller than $15 \%$ since $z \sim 0.5$.

2. The thinness and coldness of the stellar distribution in galactic disks imply that these disks 
did not encounter substantial merging since gas was converted into stars in them. Tóth \& Ostriker (1992) have obtained an upper limit of $4 \%$ on the fraction of the Galactic mass inside the solar radius that could have accreted within the last 5 billion years, based on the observed scale and Toomre $Q$-parameter of the Milky-Way disk.

3. Considerable evolution in the potential wells of spiral galaxies since $z \sim 1$ would have resulted in a considerable scatter in their Tully-Fisher relation due to variations in their formation histories. Eisenstein \& Loeb (1996) have used Monte Carlo realizations of halo formation histories in hierarchical models of structure formation to limit any substantial evolution in the potential wells of spirals since $z \sim 1$, based on the observed tightness in their Tully-Fisher relation.

4. The statistics of multiply imaged QSOs implies that galactic lenses (either ellipticals or spirals) did not encounter significant merging below $z \sim 1$ (Mao \& Kochanek 1994; Rix et al. 1994).

5. Measurements of the rotation velocities and luminosities of spiral galaxies at intermediate redshifts (Vogt et al 1996) imply that they are kinematically similar to nearby spirals.

6. Deep imaging and spectroscopy in the field of 3C 336 (Steidel et al. 1996) show a lack of strong evolution in the population of galactic metal-line absorbers up to a redshift $z \sim 1$.

For redshifts below unity, the observed evolution in $\Omega_{\mathrm{HI}}$ is also very weak (cf. Fig. 8 in Wolfe et al. 1995). Based on all of this evidence, we adopt a model in which the gravitational potentials and the HI+dust content of the local population of spiral galaxies had not evolved significantly between a redshift of $0.6 \pm 0.2$ and the present time.

The paper is organized as follows. In $\S 2$ we summarize our parameterization of dust at high redshift, focusing on its relation to neutral hydrogen in damped Ly $\alpha$ systems. We then examine in $\S 3$ the simple situation of gravitational lensing and dust obscuration of a single point source by a single lens. We turn to statistical considerations in $\S 4$, where we detail our method to calculate the combined effects of gravitational lensing and dust obscuration of a population of background QSOs by an intervening population of damped Ly $\alpha$ absorbers. Finally, $\S 5$ summarizes our conclusions.

\section{Dust Model}

Direct evidence that damped Ly $\alpha$ systems contain dust is provided by the reddening of background QSOs and by the depletion of Cr relative to Zn. The amount of dust is usually expressed in terms of the dust-to-gas ratio $k$ by

$$
k \equiv \frac{\tau_{B}}{N_{21}},
$$


where $\tau_{B}$ is the optical depth due to dust in the rest frame $B$ band and $N_{21}$ is the column density of neutral hydrogen in units of $10^{21} \mathrm{~cm}^{-2}$.

The reddening of background QSOs can be estimated by comparing the spectral indices of quasars with and without damped Ly $\alpha$ absorbers. Typical dust-to-gas ratios in the damped Ly $\alpha$ systems can then be inferred from this reddening estimate if a shape for the extinction curve is assumed.

Another way to infer $k$ is provided by measurements of the gas-phase abundances of $\mathrm{Zn}, \mathrm{Cr}$, and other trace elements. The underlying assumption is that, apart from overall scalings in the metallicity and dust-to-gas ratio, the relative composition of the interstellar medium is the same in the damped Ly $\alpha$ systems as it is in the Milky Way (i.e., the abundance patterns and grain composition are the same in both). Measuring the ratio $\mathrm{Zn} / \mathrm{H}$ and the depletion of $\mathrm{Cr}$ relative to $\mathrm{Zn}$, one can estimate the fraction of gaseous $\mathrm{Cr}$ that has condensed into dust grains. Both methods of measuring $k$ yield a typical dust-to-gas ratio for damped Ly $\alpha$ systems at $z \approx 2$ that is roughly $10 \%$ of the Milky Way value $k \approx 0.8$.

The dust-to-gas ratio evolves with redshift due to various astrophysical processes, such as conversion of gas into stars, metal enrichment and inflows or outflows of gas from galaxies. However, since we focus on low redshift absorbers, we assume no evolution in their dust-to-gas ratio,

$$
\bar{k}(z) \simeq \bar{k}(0) .
$$

The dust optical depth $\tau(z)$ in an absorber at redshift $z$ is related to the optical depth in the $B$ band $\tau_{B}$ by

$$
\tau(z)=\tau_{B} \xi\left(\frac{\lambda_{B}}{1+z}\right)
$$

where $\xi(\lambda)$ is the ratio of the extinction at wavelength $\lambda$ to that in the $B$ band. Observations (Mathis 1990; Savage \& Mathis 1979) indicate that the extinction curve declines with wavelength roughly as $\xi(\lambda) \propto \lambda^{-1}$. For wavelengths not too far from the $B$ band, a good fit is provided by

$$
\xi(\lambda) \simeq 0.4 \frac{1}{\lambda} \mu \mathrm{m} .
$$

In terms of the dust-to-gas ratio as defined in equation (11), the optical depth can be written as

$$
\tau(z)=k(z) N_{21} \xi\left(\frac{\lambda_{B}}{1+z}\right)
$$

The intrinsic flux $S^{\prime}$ of a source is changed to $S$ if it passes an intervening galaxy. Gravitational lensing magnifies the flux by a factor $\mu$, while obscuration by dust reduces the flux by a factor $\exp (-\tau)$ (Fall \& Pei 1989). The net effect is thus

$$
S=\mu \exp (-\tau) S^{\prime}
$$




\section{Combination of Lensing and Dust Obscuration}

We now examine the combined effect of dust obscuration and gravitational lensing in the simple situation of a point source which is lensed and obscured by a single intervening galaxy. For definiteness, we adopt a source redshift of $z_{\mathrm{s}}=3$ and a lens redshift of $z_{\mathrm{d}} \equiv z_{\mathrm{abs}}=0.5$ where necessary.

We describe the profile of the neutral hydrogen disk in the galaxy by the radius where the face-on column density is $N^{\prime}$,

$$
R_{\mathrm{H}}\left(N^{\prime}\right)=f_{\mathrm{H}}\left(N^{\prime}\right) R_{*} l^{t}
$$

where $l=L / L_{*}$ is the galaxy luminosity, $t \approx 0.4$, and $R_{*} \approx 11 h^{-1} \mathrm{kpc}$ is a characteristic radius of an $L_{*}$ galaxy. The data by Broeils \& van Woerden (1994) imply an exponential neutral hydrogen profile,

$$
f_{\mathrm{H}}\left(N^{\prime}\right)=\frac{3}{2}-2 \log \left(\frac{N^{\prime}}{2 \times 10^{20} \mathrm{~cm}^{-2}}\right) .
$$

For simplicity, we will in this section consider a lensing galaxy with luminosity $L_{*}$. The hydrogen radius of equation (7) then simplifies to $R_{\mathrm{H}}\left(N^{\prime}\right)=f_{\mathrm{H}}\left(N^{\prime}\right) R_{*}$, where $N^{\prime}$ is the face-on column density of HI. If the disk is inclined by an angle $\theta$ with respect to the line-of-sight, the observed column density is $N=N^{\prime} / \cos \theta$.

The intercepted HI column density not only depends on the relative position of absorber and source, but also on the lensing properties of the absorber because the light rays are bent. We model the lens as a singular isothermal sphere (SIS). Recent work (Kochanek 1996) has shown that, in the case of elliptical galaxy lenses, the addition of a small core radius, consistent with the kinematics and the image configuration of the lens, does not change the lensing probability significantly. Since the light distribution in the central bulge of spiral galaxies resembles that in elliptical galaxies, we assume the SIS model to be a good approximation for our purposes.

In the SIS model, the scaled distances of the source $y$ and of the images $x$ from the center of the lens are related by (e.g., Schneider et al. 1992)

$$
x=\left\{\begin{array}{ll}
y \pm 1 & \text { if } y \leq 1 \\
y+1 & \text { if } y>1
\end{array} .\right.
$$

The appropriate length scale for $x$ is

$$
\zeta_{0} \equiv 4 \pi\left(\frac{\sigma_{v}}{c}\right)^{2} \frac{c}{H_{0}} \frac{r_{\mathrm{d}} r_{\mathrm{ds}}}{r_{\mathrm{s}}}
$$

where $r_{\mathrm{d}, \mathrm{s}, \mathrm{ds}}$ are the angular diameter distances from the observer to the lens, to the source, and from the lens to the source, respectively, in units of the Hubble length $c H_{0}^{-1}$. In the numerical examples throughout the paper, we will use a Hubble constant $H_{0}=100 \mathrm{hm} \mathrm{s}^{-1} \mathrm{Mpc}^{-1}$, with

$h=0.5$, and an $L_{*}$ galaxy velocity dispersion of $\sigma_{v, *} \approx 160 \mathrm{~km} \mathrm{~s}^{-1}$. The magnification of a point 
source by an SIS lens is

$$
\mu= \begin{cases}2 / y & \text { if } y \leq 1 \\ 1+1 / y & \text { if } y \geq 1\end{cases}
$$

In order to compute the optical depth due to dust obscuration, we need to calculate the column density $N^{\prime}$ of the galactic HI disk where it is intercepted by the light rays from a source at position $y$. Since an inclined disk appears elliptical, the radius in the plane of the disk where the light rays pass the galaxy depends on the relative position angle between $y$ and the inclined galactic disk. For an inclination angle $\theta$, the radius averaged over position angle is

$$
\langle x\rangle=\frac{1}{2 \pi} \int_{0}^{2 \pi} d \varphi\left[(x \cos \varphi)^{2}+(x \sin \varphi / \cos \theta)^{2}\right]=\frac{2 x}{\pi \cos \theta} \mathrm{E}\left(\sin ^{2} \theta\right)
$$

with the elliptical function of the $2^{\text {nd }}$ kind $\mathrm{E}(x)$.

The HI column density $N^{\prime}$ in the disk at the average radius $\langle x\rangle$ is then found from

$$
R_{*} f_{\mathrm{H}}\left(N^{\prime}\right)=\langle x\rangle \zeta_{0}
$$

which can be solved for $N^{\prime}$ using $f_{\mathrm{H}}\left(N^{\prime}\right)$ from equation (8). The observed column density $N$ as a function of the source position $y$ is

$$
N(y)=\frac{2 \times 10^{20} \mathrm{~cm}^{-2}}{\cos \theta} 10^{3 / 4-\langle x(y)\rangle \zeta_{0} /\left(2 R_{*}\right)},
$$

with $x(y)$ given by equation (9). The optical depth can then be easily computed from equation (5). For the redshifts chosen, $z_{\mathrm{s}}=3$ and $z_{\mathrm{d}}=0.5$, for observations in the B-band, and for a cosmological model with $\Omega_{0}=0.2, \Omega_{\lambda}=0$, we find from equations (4) and (10)

$$
\zeta_{0} \approx 4 \mathrm{kpc}, \quad \xi \approx 1.4, \quad R_{*}=22 \mathrm{kpc},
$$

for $h=0.5$.

We now have all the ingredients to estimate the ratio between the observed and intrinsic fluxes, $S$ and $S^{\prime}$, related by equation (6). The resulting flux ratio $S / S^{\prime}$ is shown in Figure 1, where $S / S^{\prime}$ is plotted as a function of source position $y$ for different values of the inclination angle $\theta$ of the disk. For comparison, the solid line shows $S / S^{\prime}$ without taking dust obscuration into account. Figure 1 shows that for each inclination angle there is a source position $y_{*}$ such that magnification dominates over dimming (i.e., $S / S^{\prime}>1$ ) for $y<y_{*}$ while extinction is stronger than magnification (i.e., $S / S^{\prime}<1$ ) for $y>y_{*}$. As smaller $y$ imply smaller $x$, we find that magnification is stronger than extinction for light rays passing closer to the center of the disk.

\section{Statistics of Dust Obscuration and Lensing}

We now examine the combined effect of lensing and dust obscuration on a population of distant QSOs by a population of intervening galaxies. Damped Ly $\alpha$ systems offer one of the 
best ways of tracing the evolution of neutral hydrogen in spiral galaxies at intermediate and high redshifts. We therefore focus on the simultaneous effects of lensing and dust obscuration on the properties of neutral hydrogen which can be inferred from the damped absorption lines. In particular, we investigate the effects of dust and lensing on the column density distribution function of HI, $f(N)$, and on the estimates of the contribution of $\mathrm{HI}$ to the density of the Universe $\Omega_{\mathrm{HI}}$.

We model the column density profile of neutral hydrogen in the absorbers according to equations (7) and (8) and assume for simplicity that it is constant in time. The absorber population is described by a Schechter luminosity function,

$$
\Phi(l) d l=\Phi_{*} l^{s} \exp (-l) d l,
$$

for which we adopt the parameters appropriate for spiral galaxies (Marzke et al. 1994),

$$
\Phi_{*}=1.5 \times 10^{-1} h^{2} \mathrm{Mpc}^{-3}, \quad s=-0.81 .
$$

The source population of distant QSOs is parameterized in terms of the intrinsic number count function which can be well modeled by a broken power law (e.g., Boyle, Shanks, \& Peterson 1988; Hartwick \& Schade 1990),

$$
\frac{d N}{d S}(S)=A \times\left\{\begin{array}{ll}
S^{-\beta_{1}-1} & \text { if } S \leq S_{0} \\
S^{-\beta_{2}-1} & \text { if } S>S_{0}
\end{array},\right.
$$

where the flux $S_{0}$ at the break point corresponds to an apparent QSO magnitude of $B \approx 19.5$ and $A$ is a constant. A reasonable fit to observational data for the two branches of the power law is provided by the values $\beta_{1}=0.64$ and $\beta_{2}=2.52$ (Pei 1995).

If neither lensing nor dust obscuration are effective, the probability for a QSO to exhibit damped Ly $\alpha$ absorption with a column density $\geq N$ in its spectrum is given by (Bahcall \& Peebles 1969)

$$
P_{\mathrm{Ly} \alpha}\left(z_{1}, z_{2}, N\right)=\Phi_{*} \Gamma(1+s+2 t) \pi R_{*}^{2}\left\langle f_{\mathrm{H}}^{2}(N)\right\rangle \Delta X\left(z_{1}, z_{2}\right),
$$

where $\Delta X\left(z_{1}, z_{2}\right)$ is the absorption distance surveyed per QSO, and $\left\langle f_{\mathrm{H}}^{2}(N)\right\rangle$ is the average of (8) over all inclination angles.

If the absorption probability $P_{\mathrm{Ly} \alpha}\left(z_{1}, z_{2}, N\right)$ is measured, the column density distribution of absorbers $f(N)$ is given by

$$
f(N)=\frac{c}{H_{0}} \frac{1}{\Delta X\left(z_{1}, z_{2}\right)}\left|\frac{\partial P\left(z_{1}, z_{2}, N\right)}{\partial N}\right| .
$$

This expression is independent of the QSO redshift, the QSO flux, and the redshift interval surveyed for damped Ly $\alpha$ absorption.

On the other hand, if gravitational lensing is taken into account, the probability for damped Ly $\alpha$ absorption, and thus the column density distribution, does depend on the flux threshold 
$S$ and on the redshifts involved because of the magnification bias (Bartelmann \& Loeb 1996). Specifically,

$$
f_{\mathrm{GL}+\mathrm{dust}}\left(z_{1}, z_{2}, z_{\mathrm{s}}, N ; S\right)=\frac{c}{H_{0}} \frac{1}{\Delta X\left(z_{1}, z_{2}\right)} \int_{z_{1}}^{z_{2}} d z \tilde{f}_{\mathrm{GL}+\mathrm{dust}}\left(z, z_{s}, N ; S\right)
$$

with

$$
\tilde{f}_{\mathrm{GL}+\text { dust }}\left(z, z_{\mathrm{s}}, N ; S\right)=\frac{1}{N_{\mathrm{QSO}}(S)} \int_{0}^{\infty} d S^{\prime}\left|\frac{\partial p_{\mathrm{GL}}^{\prime}\left(z, z_{\mathrm{s}}, N ; S / S^{\prime}\right)}{\partial N}\right| \frac{d N_{\mathrm{QSO}}}{d S}\left(S^{\prime}\right)
$$

where $p_{\mathrm{GL}}^{\prime}\left(z, z_{\mathrm{s}}, N ; \mu\right)$ is the probability that a QSO at redshift $z_{\mathrm{s}}$ shows damped Ly $\alpha$ absorption with column density $\geq N$ and is magnified by a factor $\geq \mu$ by lenses in the redshift interval $d z$ around $z$ (cf. equation (2.25) in Bartelmann \& Loeb 1996).

Let us now consider the combined effect of lensing and dust obscuration. For an intrinsic source flux $S^{\prime}$, the observed flux is $S=S^{\prime} \mu \exp (-\tau)$ with $\tau(k, z)=k N_{21} \xi\left(\lambda_{B} / 1+z\right)$ (cf. eqs. (5) and (6)). Consequently, the distribution of absorbers in equation (22) becomes

$$
\begin{aligned}
\tilde{f}_{\mathrm{GL}+\text { dust }}\left(z, z_{\mathrm{s}}, S ; N\right) & =\frac{1}{N_{\mathrm{QSO}}(S)} \int_{0}^{\infty} d S^{\prime}\left|\frac{\partial p_{\mathrm{GL}}^{\prime}\left(z, z_{\mathrm{s}}, N ; S / S^{\prime} e^{-\tau}\right)}{\partial N}\right| \frac{d N_{\mathrm{QSO}}}{d S^{\prime}}\left(S^{\prime}\right) \\
& =\frac{A}{N_{\mathrm{QSO}}(S)}\left[\int_{0}^{S_{0}} d S^{\prime}\left|\frac{\partial p_{\mathrm{GL}}^{\prime}\left(z, z_{\mathrm{s}}, N ; S / S^{\prime} e^{-\tau}\right)}{\partial N}\right| S^{\prime-\beta_{1}-1}\right. \\
& \left.+\int_{S_{0}}^{\infty} d S^{\prime}\left|\frac{\partial p_{\mathrm{GL}}^{\prime}\left(z, z_{\mathrm{s}}, N ; S / S^{\prime} e^{-\tau}\right)}{\partial N}\right| S^{\prime-\beta_{2}-1}\right]
\end{aligned}
$$

where we have used equation (18). By changing variables $\tilde{S}=S^{\prime} e^{-\tau}$, equation (23) becomes

$$
\begin{aligned}
\tilde{f}_{\mathrm{GL}+\text { dust }}\left(z, z_{\mathrm{s}}, S ; N\right) & =\frac{A}{N_{\mathrm{QSO}}(S)}\left[e^{-\beta_{1} \tau} \int_{0}^{S_{0} e^{-\tau}} d \tilde{S}\left|\frac{\partial p_{\mathrm{GL}}^{\prime}\left(z, z_{\mathrm{s}}, N ; S / \tilde{S}\right)}{\partial N}\right| \tilde{S}^{-\beta_{1}-1}\right. \\
& \left.+e^{-\beta_{2} \tau} \int_{S_{0} e^{-\tau}}^{\infty} d \tilde{S}\left|\frac{\partial p_{\mathrm{GL}}^{\prime}\left(z, z_{\mathrm{s}}, N ; S / \tilde{S}\right)}{\partial N}\right| \tilde{S}^{-\beta_{2}-1}\right] .
\end{aligned}
$$

Hence, dust causes an apparent shift in the break flux of the QSO distribution function and damps the absorber distribution by a factor $\exp (-\beta \tau)$ (a discussion of the latter effect for bright QSOs can be found in Fall \& Pei 1993).

Since the value of $k$ varies considerably among different DLA systems, we allow for a spread in its values. Following Fall \& Pei (1993), we assume a log-normal form for the probability distribution of $k$,

$$
g(k)=\frac{1}{\sqrt{2 \pi} \sigma_{*} k} \exp \left[-\frac{\left(\ln k-\ln k_{*}\right)^{2}}{2 \sigma_{*}^{2}}\right],
$$


where the parameter $k_{*}$ is related to the mean $\bar{k}$ of the dust-to-gas ratio by

$$
\bar{k} \equiv \int_{0}^{\infty} d k g(k) k=k_{*} \exp \left(\frac{\sigma_{*}^{2}}{2}\right),
$$

with $\bar{k}$ given by equation (2) under the assmption that at $z=0$ the Milky Way value of $k$ reflects the true mean of the spiral galaxy population. Following the study of Fall \& Pei (1993; cf. their figure $8 \mathrm{~b}$ ), we adopt a constant value of $\sigma_{*}=1$. With the introduction of a probability distribution for $k$, equation (24) reads

$$
\begin{aligned}
\tilde{f}_{\mathrm{GL}+\text { dust }}\left(z, z_{\mathrm{s}}, N, S\right) & =\frac{A}{N_{\mathrm{QSO}}(S)} \int_{0}^{\infty} d k g(k, z) \\
& \times\left[e^{-\beta_{1} \tau(k, z)} \int_{0}^{S_{0} e^{-\tau(k, z)}} d S^{\prime} \frac{\partial p_{\mathrm{GL}}^{\prime}\left(z, z_{\mathrm{s}}, N, S / S^{\prime}\right)}{\partial N} S^{\prime-\beta_{1}-1}\right. \\
& \left.+e^{-\beta_{2} \tau(k, z)} \int_{S_{0} e^{-\tau(k, z)}}^{\infty} d S^{\prime} \frac{\partial p_{\mathrm{GL}}^{\prime}\left(z, z_{\mathrm{s}}, N, S / S^{\prime}\right)}{\partial N} S^{\prime-\beta_{2}-1}\right] .
\end{aligned}
$$

Equations (21) and (27) can be used to get the observed distribution of absorbers in the redshift interval $z_{1} \leq z_{\text {abs }} \leq z_{2}$. The results from these equations are displayed in Figure 2 for the absorber redshift interval $0.4 \leq z_{\text {abs }} \leq 0.8$. This figure shows the observed distribution of $N f(N)$ for two choices of the limiting flux $B$ and for three cosmological models, in comparison to the true distribution (solid line).

Having calculated $N f(N)$, the inferred column density of neutral hydrogen in absorbers with column density of $N_{1} \leq N \leq N_{2}$, normalized by the present critical density, is given by

$$
\Omega_{\mathrm{HI}}\left(N_{1}, N_{2}\right)=\frac{H_{0}}{c} \frac{\bar{m}}{\rho_{\mathrm{c}, 0}} \int_{N_{1}}^{N_{2}} d N N f(N)
$$

where $\bar{m}$ is the mean molecular mass per proton and $\rho_{\mathrm{c}, 0}$ is the present-day closure density,

$$
\rho_{\mathrm{c}, 0}=\frac{3 H_{0}^{2}}{8 \pi G} .
$$

Figure 3 displays the result for $\Omega_{\mathrm{HI}}$ for the absorber redshift interval $0.4 \leq z_{\mathrm{abs}} \leq 0.8$ and for the same three cosmological models as in Figure 2 .

The enhancement in the abundance of absorbers (and hence in the inferred density parameter of neutral hydrogen) is a consequence of the magnification bias due to lensing, which increases the number of QSOs above the detection threshold. Extinction by dust results in an opposite tendency. In Figure 4a we show the combined effect of these opposite tendencies as a function of the QSO magnitude limit, for low-redshift absorbers with $N \gtrsim 10^{20} \mathrm{~cm}^{-2}$. The QSO detection probability is compared to the case where neither of these two effects are taken into account. We find that for bright quasars with a limiting magnitude $B \lesssim 17$, the effect of lensing dominates. 
In Figure 4b we compare the above probability to the situation where only lensing is taken into account (cf. Bartelmann \& Loeb 1996). This figure illustrates that for low-redshift absorbers, the suppression of the lensing effect by dust is of several tens of percent. The level of suppression is higher for brighter QSOs because of the dependence of the extinction factor on the slope of the QSO number counts [cf. eq. (27)], which is steeper for brighter QSOs. Note that the suppression of the lensing probability by dust is weaker than the suppression of the lensing effect on $\Omega_{\mathrm{HI}}$ (compare Fig. 5 of Bartelmann \& Loeb (1996) to Fig. 3 in this paper). This follows from the fact that $\Omega_{\mathrm{HI}}$ is integrated with an extra factor of $N$ (cf. eq. 28), which tends to emphasize high column density systems more, and therefore enhance the suppression of lensing by dust at a given QSO magnitude limit.

\section{Conclusions}

Gravitational lensing by a spiral galaxy occurs when the line-of-sight to a background quasar passes within a few kpc from the center of the galactic disk. Since galactic disks are rich in neutral hydrogen, the quasar spectrum will likely be marked by a damped Ly $\alpha$ absorption trough at the lens redshift. Indeed, the observed $21 \mathrm{~cm}$ absorption in the spiral galaxy lens B0218+357 implies an HI column density of $4 \times 10^{21} \mathrm{~cm}^{-2}\left(T_{\mathrm{s}} / 100 \mathrm{~K}\right) /(f / 0.1)$, where $T_{\mathrm{s}}$ is the spin temperature and $f$ is the HI covering factor in this system . Bartelmann \& Loeb (1996) have shown that the efficiency of searches for gravitational lensing with sub-arcsecond splitting can be enhanced by 1-2 orders of magnitude if one focuses on the subset of bright quasars which show low-redshift $\left(z_{\text {abs }} \lesssim 0.8\right)$ strong Ly $\alpha$ absorption $\left(N \gtrsim 10^{21} \mathrm{~cm}^{-2}\right)$. The double-image signature of lensing could, in principle, be identified spectroscopically and without the need for high-resolution imaging; the absorption spectrum of the lens might show a generic double-step profile due to the superposition of the two absorption troughs of the different images. The different images pass the absorbing disk at different impact parameters and therefore have different spectral widths.

Lensing of optical sources by spiral galaxies is expected to be counteracted by dust obscuration, since the central opacity of nearby disks is considerable (see, e.g. Byun 1993; Davies et al. 1993; Byun, Freeman, \& Kylafis 1994; Jansen et al. 1994; Rix 1995). The distribution of dust in galactic disks is correlated with the distribution of neutral hydrogen, which in turn can be probed in the absorption spectra of QSOs. In this paper, we used the estimated dust-to-gas ratio of our galaxy and allowed a spread in its values to calculate the effects of dust on lensing by low redshift spiral galaxies. For absorbers with $z_{\text {abs }} \lesssim 0.8$, we have found that the relative importance of the magnification bias due to lensing and of the obscuration due to dust depends on the QSO magnitude limit and on the choice of cosmological parameters (cf. Fig. 4a). Overall, the effect of dust suppresses the detection probability of lensing by $\lesssim 55 \%$ (cf. Fig. $4 \mathrm{~b}$ ).

\footnotetext{
${ }^{1}$ The high HI column density in this lens will be directly calibrated based on the absorption spectrum of the QSO in forthcoming HST observations (Falco, Loeb, \& Bartelmann 1996).
} 
The magnification bias and the deflection of light rays due to lensing change the column density distribution of damped Ly $\alpha$ absorbers, $f(N)$. Although dust tends to suppress the distortion to $f(N)$ derived by Bartelmann \& Loeb (1996), the generic lensing peak for low-redshift absorbers with $N \sim 10^{21.5} \mathrm{~cm}^{-2}$ is still evident in Figure 2a. This peak results from the magnification bias, which raises faint QSOs above the detection threshold, thereby enhancing the probability for observing damped Ly $\alpha$ absorbers. The sharp cutoff at yet higher column densities arises because the bending of light rays due to lensing yields a minimum value for the impact parameter of the line-of-sight to the QSO through the galactic disk. For low-redshift absorbers and models with no cosmological constant we have found that the density parameter of neutral hydrogen $\Omega_{\mathrm{HI}}$, obtained by integrating $N f(N)$ over all column densities, is overestimated for QSO samples with $B \lesssim 16$ and is underestimated for fainter QSOs (cf. Fig. 3).

Since the current set of known damped $\operatorname{Ly} \alpha$ systems includes only a small number of low-redshift absorbers (see, e.g. Table 3 in Wolfe et al. (1995)), it is difficult to compare the statistical results shown in figures $2-4$ to existing data. We expect the significance of lensing relative to dust to decline at $z \gtrsim 1$, and to yield only a secondary correction to the inferred statistics of the current set of damped Ly $\alpha$ absorbers (Wolfe et al. 1995; Storrie-Lombardi et al. 1996a,b). The quantitative predictions of this paper will be tested in a straightforward manner with the advent of absorption data for larger statistical samples of QSOs with uniform selection criteria, such as the set of $\sim 10^{5}$ QSOs in the forthcoming Sloan digital sky survey (Gunn \& Knapp 1993).

The authors wish to thank an anonymous referee for insightful comments. This work was supported in part by the NASA ATP grant NAG5-3085 (for AL), and by the Sonderforschungsbereich SFB 375-95 of the Deutsche Forschungsgemeinschaft (for MB).

\section{REFERENCES}

Bahcall, J. N., \& Peebles, P. J. E. 1969, ApJ, 156, L7

Bartelmann, M. \& Loeb, A. 1996, ApJ, 457, 529

Boyle, B. J., Shanks, T., \& Peterson, B. A. 1988, MNRAS, 235, 935

Briggs, F. H., Wolfe, A. M., Liszt, H. S., Davis, M. M., \& Turner, K. L. 1989, ApJ, 341, 650

Broeils, A. H., \& van Woerden, H. 1994, A\&AS, 107, 129

Bynn, Y. I. 1993 PASP, 105, 993

Byun, Y. I., Freeman, K. C., \& Kylafis, N. D. 1994, ApJ, 432, 114

Carilli, C. L., Rupen, M. P. \& Yanny, B. 1993, ApJ, 412, L59

Davies, J. I., Philipps, S., Boyce, P. J., \& Disney, M. J. 1993, MNRAS, 260, 491

Eisenstein, D. J., \& Loeb, A. 1996, ApJ, 459, 432 
Falco, E., Loeb, A., \& Bartelmann, M. 1996, "A Search for Multiple Images of QSOs Seen Through Damped Lyman-alpha Absorbers", approved proposal for cycle-6 observations with HST

Fall, S. M. \& Pei, Y. C. 1989, ApJ, 337, 7

Fall, S. M. \& Pei, Y. C. 1993, ApJ, 402, 479

Fall, S. M. \& Pei, Y. C. 1995, in: QSO Absorption Lines, ed. G. Meylan (Heidelberg: Springer), pp. $23-34$

Fall, S. M., Pei, Y. C., \& McMahon, R. G. 1989, ApJ, 341, L5

Gunn, J. E., \& Knapp, J. 1993, Sky Surveys, ed. B. T. Soifer, ASP Conference Series \#43, 267

Hartwick, F. D. A., \& Schade, D. 1990, ARA\&A, 28, 437

Huchra, J., Gorenstein, M., Kenk, S., Shapiro, I., Smith, G., Horine, E., \& Perley, R. 1985, AJ, 90,69

Jansen, R. A., Knapen, J. H., Beckman, J. E., Peletier, R. F., \& Hes, R. 1994, MNRAS, 270, 373

Jaunsen, A., Hjorth, J. 1997, A\&A, 317L, 39

Kochanek, C. S. 1996, ApJ, in press, preprint astro-ph/9510077

Lanzetta, K. M., \& Bowen, D. V. 1992, ApJ, 391, 48

Lanzetta, K. M., Wolfe, A. M., Turnshek, D. A.,Lu, L., McMahon, R. G. \& Hazard, C. 1991, ApJS, 77, 1

Lanzetta, K. M., Wolfe, A. M. \& Turnshek, D. A., 1995, ApJ, 440, 435

Le Brun, V., Bergeron, J., Boissé, P., \& Deharveng, J. M. 1996, A\&A, in press, preprint astro-ph/9611031

Lu, L., \& Wolfe, A. M. 1994, AJ, 108, 44

Lu, L., Wolfe, A. M., Turnshek, D. A., \& Lanzetta, K. M. 1993, ApJS, 77, 1

Lu, L., Sargent, W. L. W., Barlow, T. A. 1996, ApJ, in press, preprint astro-ph/9606044

Lu, L., Sargent, W. L. W., Womble, D. S., \& Barlow, T. A. 1996, ApJ, 457, L1

Mao, S. \& Kochanek C. 1994, MNRAS, 268, 569

Marzke, R. O., Geller, M. J., Huchra, J. P., \& Corwin Jr., H. G. 1994, AJ, 108, 437

Mathis, J. S. 1990, ARA\&A, 28, 37

Meyer, D. M., \& Roth, K. C. 1990, ApJ, 363, 57

Meyer, D. M., Welty, D. E., \& York, D. G. 1989, ApJ, 343, L37

Meyer, D. M., \& York, D. G. 1992, ApJ, 399, L121

O’Dea, C. P., Baum, S. A., Stanghellini, C., Dey, A., van Breughel, W., Deustua, S., \& Smith, E. 1992, AJ, 104, 3120

Ostriker, J. P., \& Heisler, J. 1984, ApJ, 278, 1 
Patnaik, A. R., Browne, I. W. A., King, L. J., Muxlow, T. W. B., Walsh, D., \& Wilkinson, P. N. 1993, MNRAS, 261, 435

Pei, Y. C. 1995, ApJ, 438, 623

Pei, Y. C., Fall, S. M., \& Bechtold, J. 1991, ApJ, 378, 6

Perry, J. J., Watson, A. M., \& Kronberg, P. P. 1993, ApJ, 406, 407

Pettini, M., Boksenberg, A., \& Hunstead, R. W. 1990, ApJ, 348, 48

Pettini, M., Smith, L. J., Hunstead, R. W., \& King, D. L. 1994, ApJ, 426, 79

Rix, H.-W., Maoz, D., Turner, E., \& Fukugita, M. 1994, ApJ, 435, 49

Rix, H.-W., Schneider, D. P., \& Bahcall, J. N. 1992, AJ, 104,959

Savage, B. D., \& Mathis, J. S. 1979, ARA\&A, 17, 73

Schechter, P. 1976, ApJ, 203, 297

Schneider, P., Ehlers, J., \& Falco, E. E. 1992, Gravitational Lenses (Heidelberg: Springer)

Smith, L. J., Pettini, M., King, D. L., Hunstead, R. W. 1996, preprint astro-ph/9601153

Steidel, C. C., Pettini, M., Dickinson, M., \& Persson, S. E. 1994, AJ 108, 2046

Steidel, C. C., Bowen, D. V., Blades, J. C., Dickenson, M. 1995, ApJ, 440, L455

Steidel, C. C., Dickinson, M., Meyer, D. M., Adelberger, K. L., Sembach, K. R. 1996, preprint astro-ph/9610230

Storrie-Lombardi, L. J., Irwin, M. J., \& McMahon, R. G. 1996a, to appear in MNRAS, preprint astro-ph/9608146

Storrie-Lombardi, L. J., McMahan, R. G., Irwin, M. J., \& Hazard, C. 1996b, to appear in ApJS, 1 Sept. issue, preprint astro-ph/9604021

Tóth, G., \& Ostriker, J. P. 1992, ApJ, 389, 5

Turnshek, D. A., Wolfe, A. M., Lanzetta, K. M., Briggs, F. H., Cohen, R. D., Foltz, C. B., Smith, H. E., \& Wilkes, B. J. 1989, ApJ, 344, 567

Vogt, N. P., Herter, T., Haynes, M. P., \& Courteau, S., to appear in ApJ, preprint astro-ph/9604114

Welter, G. L., Perry, J. J. \& Kronberg, P. P. 1984, ApJ, 279, 19

Wolfe A. M. 1995, in: QSO Absorption Lines, ed. G. Meylan (Heidelberg:Springer), pp. 13-22

Wolfe, A. M., Turnshek, D. A., Smith, H. E. \& Cohen, R. D. 1986, ApJS, 61, 249

Wolfe, A. M., Lanzetta, K. M., \& Oren, A. L. 1992, ApJ, 388, 17

Wolfe, A. M., Turnshek, D. A., Lanzetta, K. M., \& Lu, L. 1993, ApJ, 404, 480

Wolfe, A. M., Turnshek, D. A., lanzetta, K. M., Lu, L., \& Oke, J. B. 1992, ApJ, 385, 151

Wolfe, A. M., Fan, X. M., Tytler, D., Vogt, S. S., Keane, M. J., \& Lanzetta, K. M. 1994, ApJ, 435, L101 
Fig. 1. - The ratio between the observed flux $S$ and the intrinsic QSO flux (without the absorbing lens), $S^{\prime}$, as a function of the dimensionless impact parameter $y$. The solid curve shows the ratio where only lensing is taken into account, while the other two curves consider the combined effect of lensing and dust for a face-on disk (dotted line) and a disk inclined by $70^{\circ}$ (dashed line).

Wolfe, A. M., Lanzetta, K. M., Foltz, C. B., Chaffee, F. H. 1995, ApJ, 454, 698

Zaritzky, D. \& Rix, H.-W. 1996, ApJ, in press, preprint astro-ph/9608086 
Fig. 2.- Column density distribution of damped Ly $\alpha$ absorbers per logarithmic column density interval, $N f(N)$, for samples of QSOs with flux limit $B=17$ [panel(a)] and $B=19$ [panel(b)]. The solid curve in both panels displays $N f(N)$ without the influence of gravitational lensing and dust extinction. The other three curves take into account both lensing and dust for three cosmological models characterized respectively by the parameters $\Omega_{0}=0.2, \Omega_{\lambda}=0.8$ (dotted line); $\Omega_{0}=0.2, \Omega_{\lambda}=0$ (dashed line); and $\Omega_{0}=1, \Omega_{\lambda}=0$ (dashed-dotted line).w In both panels the QSO redshift is fixed at $z_{\mathrm{s}}=3$ and the redshift interval for the absorbers is $0.4 \leq z_{\mathrm{abs}} \leq 0.8$ 
Fig. 3.- The ratio between the inferred and the true $\Omega_{\mathrm{HI}}$ for three cosmological models characterized respectively by the parameters $\Omega_{0}=0.2, \Omega_{\lambda}=0.8$ (dotted line); $\Omega_{0}=0.2, \Omega_{\lambda}=0$ (dashed line); and $\Omega_{0}=1, \Omega_{\lambda}=0$ (dashed-dotted line). 
Fig. 4. - Probability for a QSO to be detected with apparent magnitude brighter than $B$ and show damped Ly $\alpha$ absorption with column density $N>10^{20} \mathrm{~cm}^{-2}$ when lensing and dust obscuration are taken into account. The probability is normalized by its value in the absence of an absorber [panel (a)], or with only lensing taken into account [panel (b)]. In both panels, $z_{\mathrm{s}}=3,0.4 \leq z_{\mathrm{abs}} \leq 0.8$, and the three curves refer to the same models as in Figures 2 and 3 . 